Received: 25 September 2017

Accepted: 8 January 2018

Published online: 30 January 2018

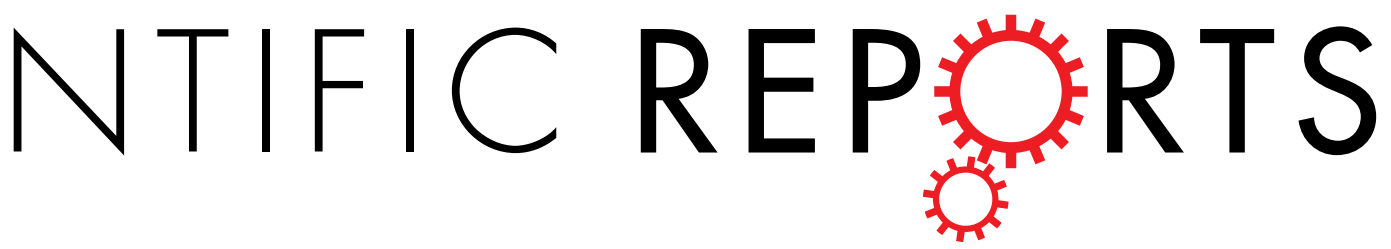

\title{
OPEN White matter hyperintensity shape and location feature analysis on brain MRI; proof of principle study in patients with diabetes
}

Jeroen de Bresser $\mathbb{1}^{1,2}$, Hugo J. Kuijf ${ }^{3}$, Karlijn Zaanen ${ }^{3}$, Max A. Viergever ${ }^{3}$, Jeroen Hendrikse ${ }^{1}$, Geert Jan Biessels ${ }^{4}$ \& Utrecht Vascular Cognitive Impairment Study Group*

Cerebral small vessel disease is a heterogeneous disease in which various underlying etiologies can lead to different types of white matter hyperintensities (WMH). WMH shape features might aid in distinguishing these different types. In this proof of principle study in patients with type 2 diabetes mellitus (T2DM), we present a novel approach to assess WMH using shape features. Our algorithm determines WMH volume and different WMH shape and location features on 3T MRI scans. These features were compared between patients with T2DM $(n=60)$ and a matched control group $(n=54)$. Although a more traditional marker (WMH volume) was not significantly different between groups (natural log transformed Beta $(95 \% \mathrm{Cl}): 0.07(-0.11 \leftrightarrow 0.24))$, patients with T2DM showed a larger number of non-punctuate WMH (median (10 ${ }^{\text {th }}-90^{\text {th }}$ percentile), patients: 40 lesions per person (16-86); controls: $26(5-58)$ ) and a different shape (eccentricity) of punctuate deep WMH (Beta (95\% Cl): 0.40 $(0.23 \leftrightarrow 0.58))$ compared to controls. In conclusion, our algorithm identified WMH features that are not part of traditional WMH assessment, but showed to be distinguishing features between patients with T2DM and controls. Future studies could address these features to further unravel the etiology and functional impact of WMH.

White matter hyperintensities (WMH) of presumed vascular origin are a key manifestation of cerebral small vessel disease (SVD) on $\mathrm{MRI}^{1}$. SVD is a heterogeneous disease in which various underlying etiologies can lead to different types of $\mathrm{WMH}^{2}$. Specific WMH features may distinguish amongst these different types. The WMH burden is currently mainly expressed in terms of volume ${ }^{1}$, but this parameter clearly lacks discriminating potential with regard to etiology. In contrast, certain location and shape features of WMH of presumed vascular origin do provide etiological and even prognostic information. For example, cerebral amyloid angiopathy (CAA) is associated with a more posterior WMH location and Cerebral Autosomal Dominant Arteriopathy with Subcortical Infarcts and Leukoencephalopathy (CADASIL) is associated with a more temporal WMH location ${ }^{3-5}$. WMH in temporal and posterior lobes are also related to an increased risk of dementia ${ }^{6}$. WMH shape and location features may also serve to differentiate WMH of presumed vascular origin from other causes of WMH, such as multiple sclerosis ${ }^{7}$. Such features (e.g. oval shape and involvement of $U$ fibers in multiple sclerosis) are used to establish the most likely etiology of WMH in clinical practice, mostly with visual assessment. However, this can be challenging and many WMH features are not readily visually perceivable. Automated detection and segmentation of WMH is preferred, but also comes with technical challenges. These challenges include partial volume effects especially for small WMH and reproducibility of measurements across MRI field strength, sequence and across variations in sequence parameters ${ }^{8}$.

Most previous studies on WMH have examined WMH volume, sometimes also addressing WMH location (e.g. $\left.{ }^{9-11}\right)$. To the best of our knowledge, WMH shape features have not been explored in depth. To study WMH

${ }^{1}$ Department of Radiology, University Medical Center Utrecht, Utrecht, The Netherlands. ${ }^{2}$ Department of Radiology, Leiden University Medical Center, Leiden, The Netherlands. ${ }^{3}$ Image Sciences Institute, University Medical Center Utrecht, Utrecht, The Netherlands. "'Department of Neurology and Neurosurgery, Brain Center Rudolf Magnus, University Medical Center Utrecht, Utrecht, The Netherlands. *A comprehensive list of consortium members appears at the end of the paper. Correspondence and requests for materials should be addressed to J.d.B. (email: J.H.J.M.de_Bresser@lumc.nl) 
shape we therefore modified an algorithm that distinguishes different shape features, which was previously used for other medical (for example in lung nodules) and non-medical applications ${ }^{12,13}$. We aimed to apply this algorithm to (1) assess different WMH shape features, (2) determine which features have the best test characteristics for the assessment of WMH shape, and (3) apply this WMH shape feature in a proof of principle study in patients with type 2 diabetes mellitus (T2DM; a condition known to be associated with an increased burden of SVD ${ }^{14}$ ) and a matched control group.

\section{Materials and Methods}

Image analysis of WMH features. Image preprocessing. Fluid attenuated inversion recovery (FLAIR) images are used for manual segmentation of WMH. This manual segmentation was performed blinded for patient status. To minimize the staircase effect of voxels across slices and to approximate the 3D shape better, a mesh is created of the manual segmentations with the marching cubes algorithm ${ }^{15}$. From the meshes, individual WMH are determined based on connectivity of the neighboring 26 voxels (3D connectivity). Then, WMH consisting of less than five voxels $(<0.014 \mathrm{ml})$ are excluded, because shape analysis cannot be accurately performed on these small lesions. The resulting meshes of the manual segmentations are used as input for the shape and location analysis and to calculate WMH volume.

WMH shape features. The WMH shape features that were determined are divided in area based (surface area), dimension/volume based (eccentricity and three measures of compactness) and complex features (fractal dimensions, shape index and curvedness) ${ }^{13}$. These features are calculated in $3 \mathrm{D}$ on the meshes of the manual segmentations as follows.

Surface area is calculated from the mesh and corrected for intracranial volume.

Eccentricity is defined as:

$$
\text { Eccentricity }=\frac{\text { diameter }_{\text {max }}}{\text { diameter }_{\text {min }}}
$$

In this definition 'diameter ${ }_{\max }$ ' denotes the largest diameter of the lesion in $3 \mathrm{D}$ and 'diameter ${ }_{\text {min }}$ ' denotes the smallest diameter of the lesion in $3 \mathrm{D}$ orthogonal to 'diameter ${ }_{\max }{ }^{13}$.

The three measures of compactness are defined as ${ }^{13}$ :

$$
\begin{gathered}
\text { Compactness } 1=\frac{\text { volume }^{2}}{\text { area }^{3}} \\
\text { Compactness } 2=\frac{\text { volume }}{\text { dim }_{x} \text { dim }_{y} \operatorname{dim}_{z}} \\
\text { Compactness } 3=\frac{\text { volume }_{\text {dim }}^{3}}{\text { max }}
\end{gathered}
$$

In these definitions 'volume' denotes the volume calculated from the mesh, 'area' is the surface area, 'dim ', ' $\operatorname{dim}_{\mathrm{y}}$ ' and ' $\mathrm{dim}_{\mathrm{z}}$ ' are the diameters along the specific axis and ' $\mathrm{dim}_{\max }$ ' is the maximum diameter along the $\mathrm{x}, \mathrm{y}$ or $\mathrm{z}$ axis.

Fractal dimensions are a measure of topological complexity and are calculated by a box counting method ${ }^{12}$.

Shape index and curvedness values are calculated for all voxels in a lesion and the median of the calculated values was taken to describe a lesion ${ }^{13}$. Shape index and curvedness are defined as:

$$
\begin{gathered}
\text { Shape index }=\frac{2}{\pi} \tan ^{-1} \frac{k_{1}+k_{2}}{k_{1}-k_{2}} \\
\text { Curvedness }=\sqrt{k_{1}^{2}+k_{2}^{2}}
\end{gathered}
$$

In these definitions ' $k_{1}$ ' and ' $k_{2}$ ' are the principal curvatures calculated using the first and second order derivative of the image blurred with a Gaussian filter with scale $\sigma=1$ voxel $^{13}$.

WMH location features. To determine location features, all WMH are transformed to MNI152 atlas space ${ }^{16}$ by registering the FLAIR images to MNI with Elastix and applying the transformation parameters to the $\mathrm{WMH}^{17}$. In MNI space, $\mathrm{WMH}$ located $>1.0 \mathrm{~cm}$ from the ventricles and with a maximum diameter of $<1.5 \mathrm{~cm}$ are automatically determined as punctuate deep WMH. These results were manually checked and in some cases corrected. Manual corrections were performed in a minority of patients and within a maximum of 2 lesions per patient. Corrections were needed mostly for segmentation errors due to for the algorithm 'unconnected parts' of the lateral ventricles, which can occur in a parietal and occipital location. In MNI space, the location of individual punctuate deep WMH was determined (frontal, temporal, parietal or occipital lobe) and recorded as a location feature.

WMH not classified as punctuate deep WMH consisted of periventricular and (early) confluent WMH and are analyzed as one group named non-punctuate WMH. The location features of non-punctuate WMH are not determined because these lesions often extend in multiple lobes. 
Subject groups. Participants. Patients with T2DM and matched controls were participants from the second Utrecht Diabetic Encephalopathy Study (UDES2) (for details see ${ }^{18,19}$ ). These participants were included through their general practitioners between April 2010 and June 2011. Inclusion criteria were that participants had to be functionally independent, between 65 and 80 years of age and Dutch-speaking. The diagnosis of T2DM was made if participants had diabetes for at least a year, were receiving treatment or had a fasting blood glucose $\geq 7.0 \mathrm{mmol} / \mathrm{L}$. Exclusion criteria were a psychiatric or neurological disorder that could influence cognitive functioning, nondisabling stroke in the past 2 years, disabling stroke, major depression, alcohol abuse or indications of dementia (mini-mental state examination score $\leq 24$ ). Control participants with a fasting blood glucose $\geq 7.0 \mathrm{mmol} / \mathrm{L}(\mathrm{n}=3)$, and participants who had severe artefacts on their brain MRI scans or an inadequate scanning protocol $(n=3)$ were excluded. The participants included in our study all had WMH (60 patients with T2DM and 54 controls). This study was approved by the medical ethics committee of the University Medical Center Utrecht and carried out in accordance with relevant guidelines and regulations. All participants signed an informed consent form.

Details regarding differences in cognitive performance and findings on regular brain MRI measures have been published previously ${ }^{20}$. In short, patients with T2DM performed slightly worse than control subjects on cognitive testing (mean differences in standardized $\mathrm{z}$ scores (95\% CI) between patients and controls: information processing speed -0.24 ( -0.58 to 0.11 ), attention and executive functioning -0.21 ( -0.50 to 0.09 ), memory -0.14 $(-0.44$ to 0.17$)$ ), but the differences were not statistically significant. Cerebral gray matter volumes were smaller (effect size $0.6, p=0.02$ ) and lateral ventricle volumes were larger (effect size $0.7, p=0.02$ ) in the patients with T2DM compared to the control subjects.

MRI scanning protocol. Brain MRI scans for all participants were acquired on a Philips 3T MRI system. A standardized protocol was used consisting of, amongst others, a FLAIR sequence (TR: $11000 \mathrm{~ms}$, TE: $125 \mathrm{~ms}$, TI: $2800 \mathrm{~ms}$, acquisition matrix: $232 \times 148$, slice thickness: $3 \mathrm{~mm}$, acquired voxel size: $0.99 \times 1.28 \times 3.00 \mathrm{~mm}^{3}$, reconstructed voxel size: $0.96 \times 0.96 \times 3.00 \mathrm{~mm}^{3}$ ), a T1 inversion recovery (IR) sequence (TR: $4416 \mathrm{~ms}$, TE: $15 \mathrm{~ms}$, TI: $400 \mathrm{~ms}$, acquisition matrix: $232 \times 185$, slice thickness: $3 \mathrm{~mm}$, acquired voxel size: $0.99 \times 1.02 \times 3.00 \mathrm{~mm}^{3}$, reconstructed voxel size: $0.96 \times 0.96 \times 3.00 \mathrm{~mm}^{3}$ ) and a 3D T1-weighted sequence (TR: $7.9 \mathrm{~ms}$, TE: $4.5 \mathrm{~ms}$, acquisition matrix: $256 \times 232$, acquired voxel size: $1.00 \times 1.00 \times 1.00 \mathrm{~mm}^{3}$, reconstructed voxel size: $1.00 \times 1.00 \times 1.00 \mathrm{~mm}^{3}$ ).

Other MRI measures. Presence of cerebral lacunar infarcts and large vessel infarcts $(>1.5 \mathrm{~cm})$ was rated visually on the FLAIR and 3D T1-weighted MRI images. Gray and white matter volumes were determined automatically on the 3D T1-weighted images by using FreeSurfer (http://surfer.nmr.mgh.harvard.edu; ${ }^{21}$ ). Intracranial volumes were manually segmented on the T1 IR images ${ }^{20}$. Gray and white matter volume were expressed as a percentage of intracranial volume.

Statistical analysis. WMH shape features (surface area, eccentricity, three measures of compactness, fractal dimensions, shape index and curvedness) were calculated for the WMH in all subjects. For these WMH features mean, minimum, maximum and skewness were calculated per lesion. Kolmogorov-Smirnov tests were performed to test for non-normal distribution.

Regarding the participant groups, differences in characteristics between the patients with T2DM and controls were assessed with T-tests for continuous variables, $\chi^{2}$ tests for proportions and Mann-Whitney U Tests for non-parametric data. WMH volumes and numbers were natural log transformed because of non-normal distribution (Kolmogorov-Smirnov; $\mathrm{p}<0.05$ ). To retain the direction of effect, the WMH volumes were first scaled to a range above 1 (multiplication by 10000). Between-group differences in WMH volume (as a percentage of intracranial volume), number and shape (median eccentricity) were assessed with linear regression analyses adjusted for age and sex. These differences were analyzed separately for all $\mathrm{WMH}$, non-punctuate $\mathrm{WMH}$ and punctuate deep WMH. To assess between-group differences in location features (frontal, temporal, parietal and occipital) of punctuate deep $\mathrm{WMH}, \chi^{2}$ tests were performed on the percentages of WMH per location. Between-group differences in punctuate deep WMH shape per location were analyzed with Mann Whitney U tests.

As a secondary analysis within the group of patients with T2DM, the association of features of all WMH (volume, number and shape (eccentricity)) with white matter volume, gray matter volume and diabetes duration was assessed with linear regression analyses adjusted for age and sex (and additionally for intracranial volume for WMH volumes).

Data availability. Anonymized imaging data are publicly available from the Utrecht Vascular Cognitive Impairment Study Group (http://www.isi.uu.nl/Research/Databases/WMHShape/). Questions can be addressed to Manja Litjens (MLitjens@umcutrecht.nl) of the Utrecht Vascular Cognitive Impairment Study Group Data Access Committee.

\section{Results}

WMH shape features. An overview of the different WMH shape features for both subject groups combined is shown in Table 1. All WMH features had a non-normal distribution (Kolmogorov-Smirnov; $\mathrm{p}<0.05)$. In this respect surface area shows the largest skewness (13.82) compared to the other WMH features $(\leq 2.20)$. Surface area, all compactness measures, eccentricity and fractal dimensions show a floor effect (the minimum measured value is close to or similar to the smallest possible value of these features). Compactness 2 and compactness 3 show a ceiling effect (the maximum measured value is close to or similar to the largest possible value of these features). This implies that variance in WMH shape might not be adequately measured by these features. The output of the complex WMH features (fractal dimensions, shape index and curvedness) proved to be difficult to comprehend and link to visual observations of WMH shape. 


\begin{tabular}{|l|l|l|l|l|l|}
\hline & Mean & Minimum & Maximum & Skewness & Kolmogorov-Smirnov \\
\hline Area based & 0.01 & 0.00 & 1.09 & 13.82 & $\mathrm{p}<0.001$ \\
\hline Surface area & ${ }^{\dagger}$ & \multicolumn{5}{l}{} \\
\hline Dimension/volume based & \multicolumn{7}{|l|}{} \\
\hline Eccentricity & 2.52 & 1.00 & 8.97 & 1.57 & $\mathrm{p}<0.001$ \\
\hline Compactness1 & 0.0039 & 0.0001 & 0.0073 & -0.10 & $\mathrm{p}<0.001$ \\
\hline Compactness2 & 0.52 & 0.03 & 1.00 & 0.25 & $\mathrm{p}<0.001$ \\
\hline Compactness3 & 0.20 & 0.00 & 1.00 & 1.14 & $\mathrm{p}<0.001$ \\
\hline Complex features & \multicolumn{7}{|l|}{} \\
\hline Fractal dimensions & 1.37 & 0.00 & 2.62 & -0.51 & $\mathrm{p}<0.001$ \\
\hline Shape index & -0.64 & -0.73 & -0.45 & 0.86 & $\mathrm{p}<0.001$ \\
\hline Curvedness & 2.30 & 0.63 & 7.71 & 2.20 & $\mathrm{p}<0.001$ \\
\hline
\end{tabular}

Table 1. Overview of different WMH shape features per lesion. Mean, minimum, maximum, skewness and output of the Kolmogorov-Smirnov test are shown for different WMH shape features. These values were calculated per lesion on the combined patient and control group. ${ }^{\dagger}$ Corrected for intracranial volume.
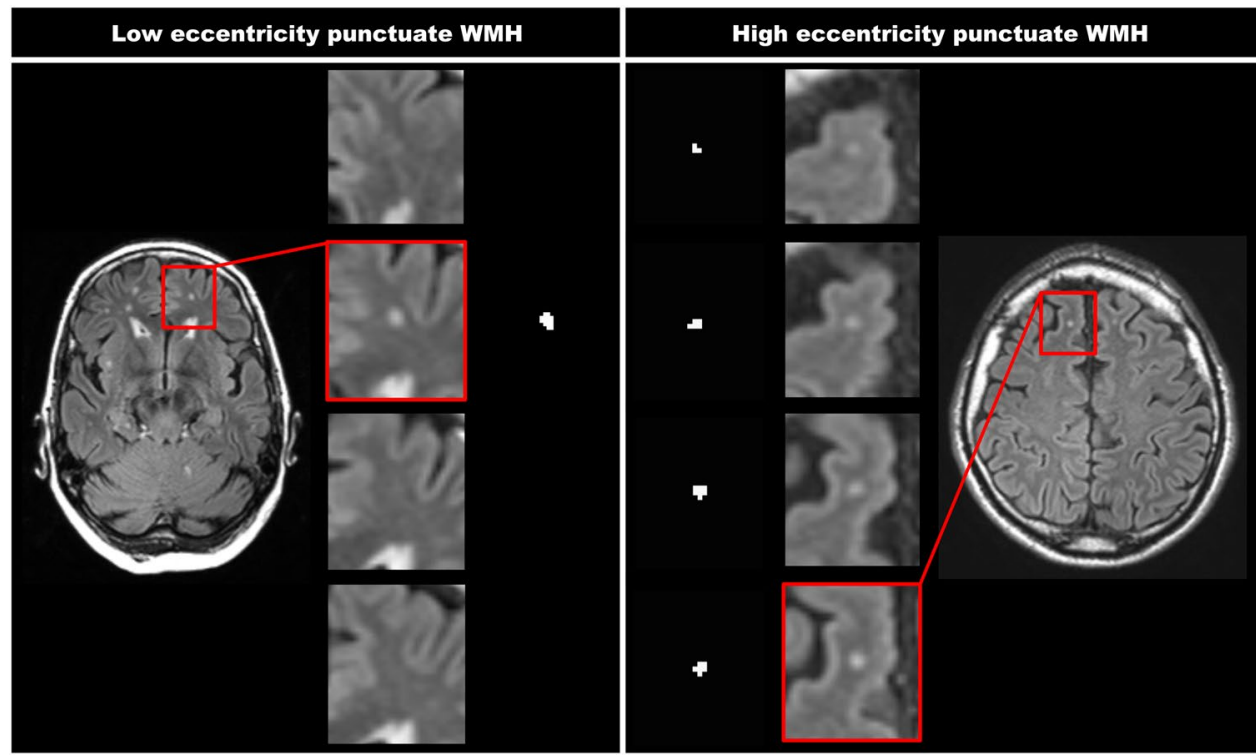

Figure 1. Two WMH with a different eccentricity value. This figure represents two WMH that have a different eccentricity value. The shown FLAIR images have a voxel size of $0.96 \times 0.96 \times 3.00 \mathrm{~mm}^{3}$. The left panels show a punctuate deep WMH with a low eccentricity of 1.0 (close to spherical), which is seen in only one slice. The right panels show a punctuate deep WMH with a high eccentricity of 4.2 (strongly ellipsoidal), which is caused by the lesion extending in multiple slices. As can be appreciated, this difference in WMH shape can also be perceived visually.

Eccentricity was chosen to test as a WMH shape feature for the between group comparisons, because it is translation-, scale- and rotation-invariant, has a relatively small skewness, does not show a ceiling effect in our measurements, has a limited floor effect (a perfect sphere) and is easy to comprehend and link to visual observations of WMH shape. An example of the WMH shape feature eccentricity for a punctuate deep WMH is shown in Fig. 1. A low eccentricity means close to spherical and a high eccentricity means strongly ellipsoidal. As can be appreciated from the figure, this difference in WMH shape can also be perceived visually.

WMH features in the subject groups. The characteristics of the group with T2DM (mean age (range): 71 years (65-80)) and the control group (71 years (66-80)) are shown in Table 2. Compared to controls, patients showed a smaller gray matter volume $(\mathrm{p}=0.009)$. White matter volume, lacunar infarcts and large vessel infarcts showed no between group differences $(\mathrm{p}>0.05)$.

$\mathrm{WMH}$ volume, number and shape (eccentricity) for all WMH, non-punctuate WMH (periventricular and (early) confluent WMH) and punctuate deep WMH are shown in Table 3; differences between patients and controls are shown as regression B coefficients (95\% CI) and regression Beta coefficients (95\% CI). Total WMH volume was similar in patients with T2DM and controls (natural log transformed (NL) Beta (95\% CI): 0.07 $(-0.11 \leftrightarrow 0.24)$ ), but the number of WMH was larger (median $\left(10^{\text {th }}-90^{\text {th }}\right.$ percentile), patients: 55 (17-123); controls: 40 (9-90)) and eccentricity of WMH was higher (Beta (95\% CI): $0.34(0.16 \leftrightarrow 0.51)$ ). 


\begin{tabular}{|c|c|c|c|}
\hline & Patients with T2DM $(n=60)$ & Control participants $(n=54)$ & p-values \\
\hline \multicolumn{4}{|l|}{ Characteristics } \\
\hline Men (\%) & $35(58)$ & $32(59)$ & 0.920 \\
\hline Age (years) & $71.0 \pm 4.3$ & $71.2 \pm 4.7$ & 0.794 \\
\hline Mean arterial pressure $(\mathrm{mmHg})^{\dagger}$ & $103 \pm 10$ & $102 \pm 11$ & 0.613 \\
\hline $\operatorname{HbAlc}(\%)^{*}$ & $6.7(5.9-7.8)$ & $5.6(5.3-6.2)$ & $<0.001$ \\
\hline Diabetes duration (years) & $10.6 \pm 8.7$ & - & - \\
\hline \multicolumn{4}{|l|}{ MRI } \\
\hline Lacunar infarcts (\%) & $20(33)$ & $17(31)$ & 0.833 \\
\hline Large vessel infarcts (\%) & $4(7)$ & $1(2)$ & 0.203 \\
\hline Gray matter volume (\%ICV) & $38.0 \pm 2.3$ & $39.1 \pm 2.1$ & 0.009 \\
\hline White matter volume (\%ICV) & $29.8 \pm 2.4$ & $30.2 \pm 3.0$ & 0.447 \\
\hline
\end{tabular}

Table 2. Characteristics of the subject groups. Data are $\mathrm{n}$ (percentage), mean $\pm \mathrm{SD}$ or median $\left(10^{\text {th }}-90^{\text {th }}\right.$ percentile). ${ }^{\dagger}$ Systolic and diastolic blood pressure were measured on three different time points. The mean arterial pressure was calculated from the averaged systolic and diastolic blood pressure. ${ }^{*}$ Determined with standardized laboratory testing. T2DM: type 2 diabetes mellitus. \%ICV: percentage of intracranial volume.

\begin{tabular}{|c|c|c|c|c|}
\hline & \multirow[b]{2}{*}{ Patients with T2DM } & \multirow[b]{2}{*}{ Control participants } & \multicolumn{2}{|c|}{ Differences between patients and controls ${ }^{\dagger}$} \\
\hline & & & B $(95 \%$ CI $)$ & Beta $(95 \% \mathrm{CI})$ \\
\hline \multicolumn{5}{|c|}{ All WMH (60 patients; 54 controls) } \\
\hline Volume (\%ICV) & $0.20(0.04-0.86)$ & $0.19(0.03-0.89)$ & $0.16(-0.27 \leftrightarrow 0.58)$ & $0.07(-0.11 \leftrightarrow 0.24)$ \\
\hline Number & $55(17-123)$ & $40(9-90)$ & $0.42(0.12 \leftrightarrow 0.71)^{*}$ & $0.25(0.07 \leftrightarrow 0.43)^{*}$ \\
\hline Shape (eccentricity) ${ }^{*}$ & $2.36 \pm 0.30$ & $2.10 \pm 0.40$ & $0.25(0.12 \leftrightarrow 0.38)^{*}$ & $0.34(0.16 \leftrightarrow 0.51)^{*}$ \\
\hline \multicolumn{5}{|c|}{ Non-punctuate WMH (60 patients; 54 controls) } \\
\hline Volume (\%ICV) & $0.19(0.04-0.82)$ & $0.18(0.03-0.80)$ & $0.27(-0.21 \leftrightarrow 0.74)$ & $0.10(-0.08 \leftrightarrow 0.28)$ \\
\hline Number & $40(16-86)$ & $26(5-58)$ & $0.62(0.33 \leftrightarrow 0.92)^{*}$ & $0.37(0.19 \leftrightarrow 0.54)^{*}$ \\
\hline Shape (eccentricity) & $2.48 \pm 0.36$ & $2.37 \pm 0.61$ & $0.10(-0.08 \leftrightarrow 0.28)$ & $0.10(-0.08 \leftrightarrow 0.29)$ \\
\hline \multicolumn{5}{|c|}{ Punctuate deep WMH (56 patients; 52 controls) } \\
\hline Volume (\%ICV) & $0.015(0.001-0.061)$ & $0.017(0.003-0.102)$ & $-0.28(-0.80 \leftrightarrow 0.24)$ & $-0.10(-0.29 \leftrightarrow 0.09)$ \\
\hline Number & $12(2-32)$ & $12(2-43)$ & $-0.11(-0.52 \leftrightarrow 0.30)$ & $-0.05(-0.24 \leftrightarrow 0.14)$ \\
\hline Shape (eccentricity) $)^{\ddagger}$ & $2.06 \pm 0.35$ & $1.79 \pm 0.25$ & $0.27(0.15 \leftrightarrow 0.39)^{*}$ & $0.40(0.23 \leftrightarrow 0.58)^{*}$ \\
\hline
\end{tabular}

Table 3. WMH features per subject. Lesion volume (median $\left(10^{\text {th }}-90^{\text {th }}\right.$ percentile)), number (median $\left(10^{\text {th }}-90^{\text {th }}\right.$ percentile $)$ and shape (mean \pm SD) of WMH are shown for patients and controls. These values are shown separately for all WMH, non-punctuate WMH (periventricular and (early) confluent WMH) and punctuate deep WMH. Differences between patients and controls are regression B coefficients (95\% CI) and regression Beta coefficients ( $95 \% \mathrm{CI}$ ); both adjusted for age and sex. ${ }^{\dagger}$ Volume and number represent natural $\log$ transformed values. For volumes, values were first scaled to a range above 1 (multiplication by 10000) to retain the direction of effect. Within groups eccentricity showed a normal distribution (Kolmogorov-Smirnov; $\mathrm{p}>0.05) .{ }^{\ddagger}$ Shape per subject represents the median eccentricity value of individual WMH. ${ }^{*} \mathrm{p}<0.01 \mathrm{~T} 2 \mathrm{DM}$ : type 2 diabetes mellitus. WMH: white matter hyperintensities. \%ICV: percentage of intracranial volume.

For non-punctuate WMH the eccentricity (Beta (95\% CI): $0.10(-0.08 \leftrightarrow 0.29))$ and volume (NL Beta (95\% CI): $0.10(-0.08 \leftrightarrow 0.28)$ ) of WMH was similar in both groups, but patients showed a larger number of WMH (median $\left(10^{\text {th }}-90^{\text {th }}\right.$ percentile), patients: 40 (16-86); controls: 26 (5-58)). This implicates that patients had more non-punctuate WMH than controls, but the mean volume per lesion was smaller.

For punctuate deep WMH the volume (NL Beta (95\% CI): $-0.10(-0.29 \leftrightarrow 0.09))$ and number (median $\left(10^{\text {th }}\right.$ $90^{\text {th }}$ percentile), patients: $12(2-32)$; controls: $\left.12(2-43)\right)$ of WMH were similar between patients and controls, but WMH in patients had a higher eccentricity (Beta (95\% CI): $0.40(0.23 \leftrightarrow 0.58)$ ). These group differences between the eccentricity of punctuate deep WMH can be observed in Fig. 2.

For punctuate deep WMH it is also possible to determine the number and eccentricity of WMH per lobe (see Table 4). In the patient group a total of $594 \mathrm{WMH}$ were located in a frontal location, 46 in a temporal location, 213 in a parietal location and 13 in an occipital location. The distribution across lobes was similar for patients and controls (all $\mathrm{p}>0.05$ ). Compared to controls, the patients had a higher eccentricity of WMH in a frontal and parietal location $(\mathrm{p}<0.05)$. These results are graphically visualized in Fig. 3. This figure shows combined mean eccentricity maps of the punctuate deep WMH for the group of patients with T2DM as well as for the control group. This figure illustrates visually that most punctuate deep WMH were in a frontal and parietal location.

Within the group of patients with T2DM, no significant associations were found between white matter volume, gray matter volume and diabetes duration on one side and features of all WMH (volume, number and shape (eccentricity)) on the other side ( $\mathrm{p}>0.05)$. 


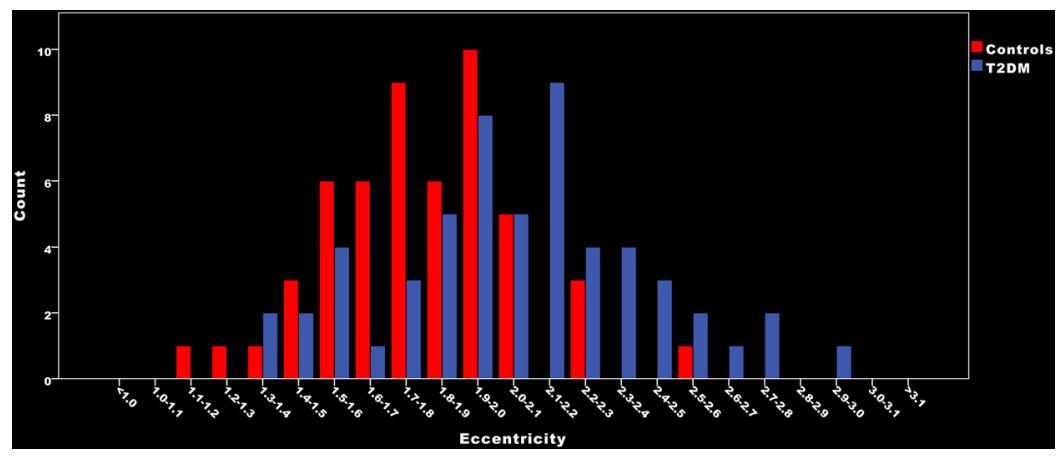

Figure 2. Median eccentricity of punctuate deep WMH per participant.

\begin{tabular}{|l|l|l|c|}
\hline \multicolumn{2}{|l|}{$\begin{array}{l}\text { Punctuate deep WMH in } \\
\text { patients with T2DM (n=866) }\end{array}$} & $\begin{array}{l}\text { Punctuate deep WMH in } \\
\text { control participants (n=881) }\end{array}$ & p-values \\
\hline \multicolumn{2}{|l|}{ Location (number) } & $561(64 \%)$ & 0.550 \\
\hline Frontal & $594(68 \%)$ & $74(8 \%)$ & 0.390 \\
\hline Temporal & $46(5 \%)$ & $234(27 \%)$ & 0.747 \\
\hline Parietal & $213(25 \%)$ & $12(1 \%)$ & 0.561 \\
\hline Occipital & $13(2 \%)$ & $1.81(1.30-2.64)$ & \\
\hline Shape (eccentricity) & $1.66(1.27-2.50)$ & 0.001 \\
\hline Frontal & $2.06(1.40-3.06)$ & $1.81(1.35-2.75)$ & 0.001 \\
\hline Temporal & $1.86(1.35-2.96)$ & $1.78(1.33-2.79)$ & 0.769 \\
\hline Parietal & $1.99(1.38-2.99)$ & & \\
\hline Occipital & $2.00(1.32-2.84)$ & & \\
\hline
\end{tabular}

Table 4. WMH shape and location features per lesion for punctuate deep WMH. Location (n (percentage)) and shape (median $\left(10^{\text {th }}-90^{\text {th }}\right.$ percentile) $)$ are shown per lesion for punctuate deep WMH. For location, $\chi^{2}$ tests were performed on the percentages. For shape, Mann Whitney $U$ tests were performed on the eccentricity values. Because of the limited sample size the $17 \mathrm{WMH}$ located in the basal ganglia region and cerebellum are not presented in this table. T2DM: type 2 diabetes mellitus. WMH: white matter hyperintensities.

\section{Discussion}

Our algorithm provides assessment of WMH volume, location and shape features, including surface area, eccentricity, different compactness measures, fractal dimensions, shape index and curvedness. Eccentricity was chosen for the proof of principle study because of favorable test characteristics. In this study, patients with T2DM did not differ from controls on traditional WMH measures (total WMH volume), but patients had more non-punctuate $\mathrm{WMH}$ and a difference in shape (eccentricity) of punctuate deep WMH compared to controls.

WMH features. WMH of presumed vascular origin are a key MRI manifestation of SVD ${ }^{1}$. Total WMH volume is the feature of WMH of presumed vascular origin that is studied most frequently $\left(\right.$ e.g. $\left.{ }^{22-29}\right)$. Few studies have also added some detail on location features (e.g. $\left.{ }^{9-11}\right)$. WMH shape features have, to our knowledge, not been explored in depth. We are also the first to provide assessment of WMH volume, location features as well as shape features. With this approach, we identified WMH features that are not part of a traditional WMH assessment, but proved to differ between patients with T2DM and controls. This study thus shows proof of principle that WMH features provide novel perspectives on cerebral SVD. Importantly, extremes of WMH shape, as identified through the algorithm, can also be perceived visually (see Fig. 1).

Neuropathology versus MRI of WMH. Neuropathological studies of WMH of presumed vascular origin showed heterogeneous underlying abnormalities ${ }^{30-32}$. The two main pathological types of WMH are abnormal white matter areas (consisting of edema) around widened venules without ischemic changes and arteriopathy with ischemic changes ${ }^{30,32}$. Smooth periventricular WMH on MRI appear to be non-vascular in origin on pathology and are considered normal anatomical structures ${ }^{32}$. On the other hand, punctuate and (early) confluent WMH on MRI showed heterogeneous underlying pathological changes ${ }^{32}$. Confluent WMH are generally considered related to underlying ischemic changes, while punctuate deep WMH are generally considered non ischemic $^{32}$. However, at some point punctuate deep WMH are starting to progress towards ischemic changes/confluency. This can sometimes be observed on brain MRI as a focal area of acute ischemia next to a punctuate deep $\mathrm{WMH}$, which precedes the occurrence of more extensive WMH in the same area ${ }^{1,2}$. Regarding our observations on MRI, possibly the first quantifiable step towards these ischemic changes/confluency is change of punctuate deep WMH to a more ellipsoidal shape (which is the dominant shape characteristic of early confluent WMH). 


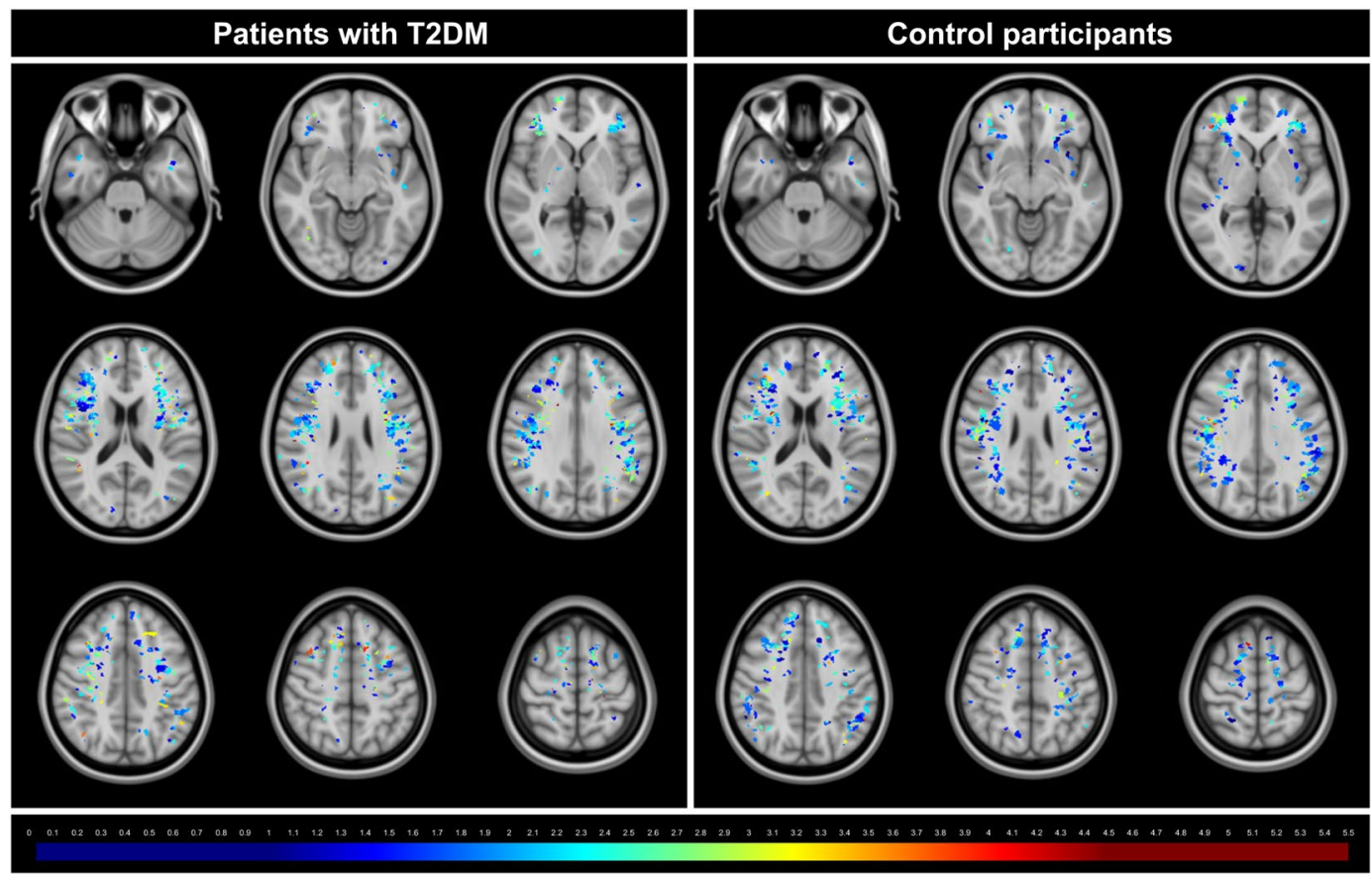

Figure 3. Mean eccentricity maps of the punctuate deep WMH. This figure illustrates mean eccentricity maps of the punctuate deep WMH for the group of patients with type 2 diabetes mellitus (T2DM) as well as for the control group. Each colored voxel represents presence of a WMH on that location in at least one participant and the color itself represents the mean eccentricity of all WMH on that location. The colors range from dark blue (low mean eccentricity) to dark red (high mean eccentricity). This figure illustrates that most punctuate deep $\mathrm{WMH}$ were in a frontal and parietal location. It also illustrates that in a frontal and parietal location there are visually less dark blue WMH in the patient group compared to the control group. These maps were obtained by automatic registration of the punctuate deep WMH to MNI152 atlas space ${ }^{16}$. Then, voxels with WMH were assigned to their respective eccentricity value ( 0 for non-lesion voxels). In both groups the eccentricity values were summed per voxel and divided by the lesion count per voxel, to obtain average eccentricity values per lesion-voxel. Due to minor registration errors some lesions are shown in cortical gray matter on the template image. This had no effect on our statistical analyses, as this template registration was only performed for the current figure.

Strengths and limitations. The strength of our study is that our algorithm provides assessment of WMH volume, location features as well as shape features. For our proof of principle study we have focused on eccentricity, because of favorable test characteristics (mainly because it is an easy to understand 3D WMH shape feature that is translation-, scale- and rotation-invariant). We have also shown the versatility of our method, because within the same framework also other WMH features can be determined (like surface area, measures of compactness, fractal dimensions, shape index and curvedness) ${ }^{13}$. A limitation of our method could be that it is dependent on accurate segmentations of all WMH. Of note, most automated WMH segmentation methods have a relatively lower accuracy compared to methods for segmentation of other brain structures ${ }^{33-35}$. Automated WMH segmentation methods also have a tendency to undersegment peripherally located punctuate deep WMH. In contrast, voxels that are erroneously segmented as WMH usually only have a limited effect on WMH volume, but could have a larger effect on WMH shape features especially in patients with a low WMH burden. We therefore chose to use the reference standard of manual segmentation of the $\mathrm{WMH}$, but this approach is clearly labor intensive. For future automation of our method, an improvement of current WMH segmentation methods will be crucial to be able to accurately assess all WMH. Another limitation of our study could be the use of 2D multi-slice FLAIR images with anisotropic voxels. The use of 3D FLAIR images with isotropic voxels will further increase the accuracy of WMH segmentations. This will result in a higher precision and accuracy of our WMH shape features. Despite this limitation, we were able to find between group differences with the use of our 2D FLAIR images.

Future implementations. Shape features of WMH of presumed vascular origin can provide additional information regarding etiology and possibly prognosis ${ }^{3-6}$. The algorithm might also be useful in distinguishing between $\mathrm{WMH}$ of presumed vascular origin and $\mathrm{WMH}$ of other etiology, for example of demyelinating origin ${ }^{36}$. Our findings show that analysis of WMH shape and location features can identify differences between patient groups that cannot readily be perceived by the naked eye. Of note, our proof of principle study was not designed to identify the exact pathophysiological processes underlying different WMH features. This should be explored in further studies. 


\section{References}

1. Wardlaw, J. M. et al. Neuroimaging standards for research into small vessel disease and its contribution to ageing and neurodegeneration. Lancet Neurol 12, 822-838, https://doi.org/10.1016/S1474-4422(13)70124-8 (2013).

2. Wardlaw, J. M., Smith, C. \& Dichgans, M. Mechanisms of sporadic cerebral small vessel disease: insights from neuroimaging. Lancet Neurol 12, 483-497, https://doi.org/10.1016/S1474-4422(13)70060-7 (2013).

3. Artero, S. et al. Neuroanatomical localisation and clinical correlates of white matter lesions in the elderly. J Neurol Neurosurg Psychiatry 75, 1304-1308, https://doi.org/10.1136/jnnp.2003.023713 (2004).

4. Thanprasertsuk, S. et al. Posterior white matter disease distribution as a predictor of amyloid angiopathy. Neurology 83, 794-800, https://doi.org/10.1212/WNL.0000000000000732 (2014).

5. Zhu, Y. C. et al. Distribution of white matter hyperintensity in cerebral hemorrhage and healthy aging. J Neurol 259, 530-536, https://doi.org/10.1007/s00415-011-6218-3 (2012).

6. Mortamais, M. et al. Spatial distribution of cerebral white matter lesions predicts progression to mild cognitive impairment and dementia. PloS one 8, e56972, https://doi.org/10.1371/journal.pone.0056972 (2013).

7. Barkhof, F. \& Scheltens, P. Imaging of white matter lesions. Cerebrovasc Dis doi:49146. 13 (Suppl 2), 21-30 (2002).

8. De Guio, F. et al. Reproducibility and variability of quantitative magnetic resonance imaging markers in cerebral small vessel disease. J Cereb Blood Flow Metab 36, 1319-1337, https://doi.org/10.1177/0271678X16647396 (2016).

9. van der Lijn, F. et al. Automated measurement of local white matter lesion volume. Neuroimage 59, 3901-3908, https://doi. org/10.1016/j.neuroimage.2011.11.021 (2012).

10. Wen, W. \& Sachdev, P. The topography of white matter hyperintensities on brain MRI in healthy 60- to 64-year-old individuals. Neuroimage 22, 144-154, https://doi.org/10.1016/j.neuroimage.2003.12.027 (2004).

11. Wen, W. \& Sachdev, P. S. Extent and distribution of white matter hyperintensities in stroke patients: the Sydney Stroke Study. Stroke 35, 2813-2819, https://doi.org/10.1161/01.STR.0000147034.25760.3d (2004).

12. Esteban, F. J. et al. Fractal dimension analysis of grey matter in multiple sclerosis. J Neurol Sci 282, 67-71, https://doi.org/10.1016/j. jns.2008.12.023 (2009).

13. Murphy, K. et al. A large-scale evaluation of automatic pulmonary nodule detection in chest CT using local image features and k-nearest-neighbour classification. Med Image Anal 13, 757-770, https://doi.org/10.1016/j.media.2009.07.001 (2009).

14. Brundel, M., Kappelle, L. J. \& Biessels, G. J. Brain imaging in type 2 diabetes. European neuropsychopharmacology: the journal of the European College of Neuropsychopharmacology 24, 1967-1981, https://doi.org/10.1016/j.euroneuro.2014.01.023 (2014).

15. Lorensen, W. E. \& Cline, H. E. Marching cubes: A high resolution 3D surface construction algorithm. Computer Graphics 21, 163-169, https://doi.org/10.1145/37401.37422 (1987).

16. Fonov, V. S., Evans, A. C., McKinstry, R. C., Almli, C. R. \& Collins, D. L. Unbiased nonlinear average age-appropriate brain templates from birth to adulthood. Neuroimage 47, S102 (2009).

17. Klein, S., Staring, M., Murphy, K., Viergever, M. A. \& Pluim, J. P. Elastix: a toolbox for intensity-based medical image registration. IEEE Trans Med Imaging 29, 196-205, https://doi.org/10.1109/TMI.2009.2035616 (2010).

18. Reijmer, Y. D. et al. Microstructural white matter abnormalities and cognitive functioning in type 2 diabetes: a diffusion tensor imaging study. Diabetes Care 36, 137-144, https://doi.org/10.2337/dc12-0493 (2013).

19. Reijmer, Y. D. et al. Disruption of the cerebral white matter network is related to slowing of information processing speed in patients with type 2 diabetes. Diabetes 62, 2112-2115, https://doi.org/10.2337/db12-1644 (2013).

20. Brundel, M. et al. Cerebral microvascular lesions on high-resolution 7-Tesla MRI in patients with type 2 diabetes. Diabetes 63, 3523-3529, https://doi.org/10.2337/db14-0122 (2014).

21. Fischl, B. FreeSurfer. Neuroimage 62, 774-781, https://doi.org/10.1016/j.neuroimage.2012.01.021 (2012).

22. de Bresser, J. et al. Progression of cerebral atrophy and white matter hyperintensities in patients with type 2 diabetes. Diabetes Care 33, 1309-1314, https://doi.org/10.2337/dc09-1923 (2010).

23. Falvey, C. M. et al. Macro- and microstructural magnetic resonance imaging indices associated with diabetes among communitydwelling older adults. Diabetes Care 36, 677-682, https://doi.org/10.2337/dc12-0814 (2013).

24. Moran, C. et al. Brain atrophy in type 2 diabetes: regional distribution and influence on cognition. Diabetes Care 36, 4036-4042, https://doi.org/10.2337/dc13-0143 (2013).

25. van Elderen, S. G. et al. Progression of brain atrophy and cognitive decline in diabetes mellitus: a 3-year follow-up. Neurology 75 , 997-1002, https://doi.org/10.1212/WNL.0b013e3181f25f06 (2010).

26. de Bresser, J. et al. Microvascular determinants of cognitive decline and brain volume change in elderly patients with type 2 diabetes. Dement Geriatr Cogn Disord 30, 381-386, https://doi.org/10.1159/000321354 (2010).

27. Espeland, M. A. et al. Influence of type 2 diabetes on brain volumes and changes in brain volumes: results from the Women's Health Initiative Magnetic Resonance Imaging studies. Diabetes Care 36, 90-97, https://doi.org/10.2337/dc12-0555 (2013).

28. Kooistra, M. et al. Diabetes mellitus and progression of vascular brain lesions and brain atrophy in patients with symptomatic atherosclerotic disease. The SMART-MR study. J Neurol Sci 332, 69-74, https://doi.org/10.1016/j.jns.2013.06.019 (2013).

29. Qiu, C. et al. Diabetes, markers of brain pathology and cognitive function: the Age, Gene/Environment Susceptibility-Reykjavik Study. Ann Neurol 75, 138-146, https://doi.org/10.1002/ana.24063 (2014).

30. Black, S., Gao, F. \& Bilbao, J. Understanding white matter disease: imaging-pathological correlations in vascular cognitive impairment. Stroke 40, S48-52, https://doi.org/10.1161/STROKEAHA.108.537704 (2009).

31. Erten-Lyons, D. et al. Neuropathologic basis of white matter hyperintensity accumulation with advanced age. Neurology 81 , 977-983, https://doi.org/10.1212/WNL.0b013e3182a43e45 (2013).

32. Schmidt, R. et al. Heterogeneity in age-related white matter changes. Acta Neuropathol 122, 171-185, https://doi.org/10.1007/ s00401-011-0851-x (2011).

33. Anbeek, P., Vincken, K. L., van Bochove, G. S., van Osch, M. J. \& van der Grond, J. Probabilistic segmentation of brain tissue in MR imaging. Neuroimage 27, 795-804, https://doi.org/10.1016/j.neuroimage.2005.05.046 (2005).

34. de Bresser, J. et al. A comparison of MR based segmentation methods for measuring brain atrophy progression. Neuroimage 54, 760-768, https://doi.org/10.1016/j.neuroimage.2010.09.060 (2011).

35. de Bresser, J. et al. Quantification of cerebral volumes on MRI 6 months after aneurysmal subarachnoid hemorrhage. Stroke 43, 2782-2784, https://doi.org/10.1161/STROKEAHA.112.669184 (2012).

36. Kanekar, S. \& Devgun, P. A pattern approach to focal white matter hyperintensities on magnetic resonance imaging. Radiologic clinics of North America 52, 241-261, https://doi.org/10.1016/j.rcl.2013.11.010 (2014).

\section{Acknowledgements}

The research of G.J.B. is supported by Vici Grant 918.16.616 from the Netherlands Organisation for Scientific Research (NWO). 


\section{Author Contributions}

J.B., J.H. and G.J.B. were involved in conceptualization; J.B., H.J.K. and K.Z. were involved in data curation; J.B. and H.J.K. were involved in formal analysis; M.A.V., J.H. and G.J.B. were involved in supervision; J.B. was involved in funding acquisition and wrote the original draft; all authors were involved in reviewing and editing the manuscript.

\section{Additional Information}

Supplementary information accompanies this paper at https://doi.org/10.1038/s41598-018-20084-y.

Competing Interests: The authors declare that they have no competing interests.

Publisher's note: Springer Nature remains neutral with regard to jurisdictional claims in published maps and institutional affiliations.

(1) Open Access This article is licensed under a Creative Commons Attribution 4.0 International License, which permits use, sharing, adaptation, distribution and reproduction in any medium or format, as long as you give appropriate credit to the original author(s) and the source, provide a link to the Creative Commons license, and indicate if changes were made. The images or other third party material in this article are included in the article's Creative Commons license, unless indicated otherwise in a credit line to the material. If material is not included in the article's Creative Commons license and your intended use is not permitted by statutory regulation or exceeds the permitted use, you will need to obtain permission directly from the copyright holder. To view a copy of this license, visit http://creativecommons.org/licenses/by/4.0/.

(c) The Author(s) 2018 


\section{Consortia}

Utrecht Vascular Cognitive Impairment Study Group

A. Algra ${ }^{5}$, E. van den Berg ${ }^{4}$, W. Bouvy ${ }^{4}$, M. Brundel ${ }^{4}$, S. Heringa ${ }^{4}$, L. J. Kappelle ${ }^{4}$, A. Leemans ${ }^{3}$, P. R. Luijten ${ }^{1}$, W. P. Th. M. Mali ${ }^{1}$, G. E. H. M. Rutten ${ }^{5}$, K. L. Vincken ${ }^{3} \&$ J. Zwanenburg ${ }^{1}$

${ }^{5}$ Julius Center for Health Sciences and Primary Care, University Medical Center Utrecht, Utrecht, The Netherlands. 